\title{
Cosmic Ray Energetics And Mass: from balloons to the ISS
}

\author{
E. S. Seo ${ }^{1}$ (for the ISS-CREAM Collaboration ${ }^{2}$ ) \\ University of Maryland \\ College Park, MD 20742, USA \\ E-mail: seo@umd.edu
}

The balloon-borne Cosmic Ray Energetics And Mass (CREAM) experiment was flown for -161 days in six flights over Antarctica. Elemental spectra were measured for $\mathrm{Z}=1$ - 26 nuclei over a wide energy range from $\sim 10^{10}$ to $>10^{14} \mathrm{eV}$ at an average altitude of $\sim 38.5 \mathrm{~km}$ with $\sim 3.9 \mathrm{~g} / \mathrm{cm}^{2}$ atmospheric overburden. Building on the success of the balloon flights, the payload has been reconfigured for exposure on the International Space Station (ISS). The ISS-CREAM instrument is configured with the CREAM calorimeter for energy measurements and four finely segmented Silicon Charge Detector layers for precise charge measurements. In addition, the Top and Bottom Counting Detectors (TCD and BCD) and Boronated Scintillator Detector (BSD) have been newly developed. The TCD and BCD are scintillator based segmented detectors to separate electrons from nuclei using their shower profile differences, while the BSD distinguishes electrons from nuclei by detecting thermal neutrons that are dominant in nuclei induced showers. The ISS-CREAM payload is currently being integrated. After system level qualification tests including Electromagnetic Interference/Compatibility (EMI/EMC), vibration, and thermal vacuum tests, the payload will be launched from NASA Kennedy Space Center on SpaceX to be installed on the ISS Japanese Experiment Module Exposed Facility. An order of magnitude increase in data collecting power is possible by utilizing the ISS to reach the highest energies practical with direct measurements. The project status and future plans will be presented.

The 34th International Cosmic Ray Conference

30 July- 6 August, 2015

The Hague, The Netherlands

\footnotetext{
${ }^{1}$ Speaker

${ }^{2}$ http://cosmicray.umd.edu/iss-cream/collaboration 


\section{Introduction}

Precise measurements of the energy spectra of elements from proton to iron in the energy range from $10^{12} \mathrm{eV}$ to $\sim 10^{15} \mathrm{eV}$ will address long-standing fundamental science questions: (1) What is the origin of the "knee" in the cosmic ray all-particle energy spectrum? (2) Can the energy spectra of cosmic rays result from a single mechanism? (3) Do supernovae really supply the bulk of cosmic rays? (4) What is the history of cosmic rays in the Galaxy? No space mission capable of measuring low particle fluxes with the needed precision at energies approaching the "knee" has yet been flown, although the Advanced Cosmic-ray Composition Experiment for the Space Station (ACCESS) was given high priority in the 2001 Decadal Study Report [1].

Cosmic Ray Energetics And Mass (CREAM) started as a balloon-borne experiment to extend direct measurements of cosmic rays to the highest energy practical. CREAM had six successful flights over Antarctica, CREAM-I to CREAM-VI, between 2004 and 2010, and it accumulated $~ 161$ days of flight time, the longest known exposure for a single balloon project. Building on the success of the balloon flights, the payload has been transformed for accommodation on the International Space Station (ISS). This ISS version of CREAM, dubbed ISS-CREAM would achieve primary science goals of the ACCESS mission, specifically it would (1) determine how the observed spectral differences of protons and heavier nuclei evolve at higher energies approaching the "knee"; (2) be capable of measuring potential changes in the spectra of secondary nuclei resulting from the interactions of primary cosmic rays with the interstellar medium; (3) conduct a sensitive search for spectral features, such as a bend in the proton spectrum; and (4) measure electrons with sufficient accuracy to determine whether or not a nearby cosmic source exists.

\section{The balloon-borne CREAM Instrument}

The CREAM instrument [2] was designed to meet the challenging requirement to have a large enough geometry factor to collect adequate statistics for the low flux of high-energy particles, and yet stay within the weight limit for balloon flights. The CREAM experiment has used complementary and redundant particle detectors to determine the charge and energy of highenergy cosmic rays from protons to iron with excellent resolution [3].

The calorimeter (CAL) combines $0.5 \lambda_{\text {int }}$ thick graphite targets and a stack of 20 tungsten plates, each $50 \mathrm{~cm} \times 50 \mathrm{~cm} \times 3.5 \mathrm{~mm}\left(1 \mathrm{X}_{0}\right)$ thick, followed by a layer of $0.5 \mathrm{~mm}$ diameter scintillating fibers grouped into fifty $1 \mathrm{~cm}$-wide ribbons. The carbon target induces hadronic interactions so showers develop in the CAL. Energy deposition in the CAL determines the particle energy and provides tracking information to determine which segment(s) of the charge detectors to use for the charge measurement. Tracking for showers is accomplished by extrapolating each shower axis back to the charge detectors.

The charge detectors are segmented to minimize hits of accompanying backscattered particles in the same segment as the incident particle. The Silicon Charge Detector (SCD) is comprised of an array of DC-type silicon PIN diodes. A cosmic ray passing through the sensor produces ionization in the depleted region that is proportional to the square of the particle charge. The building block of the SCD is a silicon sensor fabricated on a 5 inch, $380 \mu \mathrm{m}$ thick wafer. The sensor is segmented into a 4 x 4 matrix of 16 pixels. The $2.12 \mathrm{~cm}^{2}$ active area of each pixel is optimized to reduce the effect of backscatter from showers in the calorimeter, while keeping the 
channel count and power at manageable levels. The single layer SCD consists of 26 ladders, each holding seven silicon sensor modules with associated analog readout electronics to cover $79 \times 79$ $\mathrm{cm}^{2}$ area. Each element from $\mathrm{Z}=1$ to 28 are clearly separated in the single layer SCD with its $\sigma$ $\sim 0$.2e charge resolution and good linearity [3].

The exceptional performance of both the science instrument and flight support systems can be attributed to the fact that they were developed for 100-day ULDB missions. The same payload cannot be flown in consecutive years due to the time required for recovery, return to the laboratory, and refurbishment. Therefore, multiple copies of detectors were constructed to take advantage of annual flight opportunities as they become available. While the fundamental measurement principle is kept the same, significant improvements have been made over the years. Some highlights of each flight configuration are summarized below:

CREAM-I: The particle energy was measured independently by both a Transition Radiation Detector (TRD) and an ionization calorimeter. The TRD determines the Lorentz factor for $\mathrm{Z}>3$ nuclei by measuring transition $\mathrm{x}$-rays using thin-wall gas tubes. The TRD and calorimeter, which can also measure the energy of protons and He, have different systematic biases in determining particle energy. The use of both instruments allows in-flight cross-calibration of the two techniques and, consequently, provides a powerful method for measuring cosmic-ray energies.

CREAM-II: An improvement for CREAM-II and subsequent flights over CREAM-I was a dual-layer SCD, which consists of a total of 4,992 pixels. Excellent charge resolution was obtained by requiring consistency between the two charge measurements [4].

CREAM-III: A significant upgrade for CREAM-III was the addition of a Cherenkov imager (CherCam) optimized for charge measurements [5]. It consists of a silica aerogel Cherenkov radiator plane and a photon detector plane with an array of 16001 - inch diameter photomultiplier tubes (PMT's). The planes are separated by a $10 \mathrm{~cm}$ ring expansion gap to ensure that most Cherenkov photons are collected in 8 tubes surrounding the tube hit by the incident particle. Since upward moving particles will be absorbed in the radiator, the CherCam will provide efficient discrimination against backscattered particles. With CherCam, in addition to the Timing Charge Detector (TCD) based on timing, and the SCD based on pixelation, the CREAM-III instrument implements virtually all possible techniques to minimize the effect of backscatter on charge measurements in the presence of the calorimeter. In addition, the energy threshold was significantly lower than the previous two flights, due to improved CAL readout electronics [6].

CREAM-IV: An upgrade of the calorimeter readout boxes was made by providing a high voltage power supply for each two hybrid photo diodes (HPD's) instead of for each 5 HPD's. This modification was made to improve the "graceful degradation" of the calorimeter readout should HV problems occur in flight. Another improvement is a recoverable pallet. Using two halves of the CREAM-I and CREAM-II pallets, a CREAM-IV pallet was constructed using a piano hinge concept. This allows the recovered pallet to go through the Twin Otter door and be re-flyable through simple reassembly, as long as damage is not severe.

CREAM-V: To mitigate the risk of damaging or losing the Command and Data-handling Module (CDM), the CREAM Data Acquisition (CDAQ) system was modified to accommodate the Support Instrumentation Package (SIP) normally used by the Columbia Scientific Balloon Facility (CSBF) to support Long Duration Balloon (LDB) payloads. The CREAM-I to -IV instruments were supported with the CDM developed by the NASA Goddard Space Flight Center (GSFC) Wallops Flight Facility (WFF) for Ultra Long Duration Balloon (ULDB) flights. The 
CDM was nearing the end of its useful lifetime with-out a spare. The main difference between the CDM and SIP from the instrument interface viewpoint is that the SIP is serial-based, whereas the CDM was ethernet-based. The modified CDAQ with the serial interface was successfully used in both CREAM-V and CREAM-VI.

CREAM-VI: The flight duration was unusually short due to its mysterious premature termination, but the instrument recovery was the best requiring minimum repair. The calorimeter, SCD and electronics boxes on the pallet were recovered as one piece without any disassembly. Although the payload landed in a remote area and was dragged $\sim 400 \mathrm{~m}$ after impact due to late parachute separation, the science instrument was recovered without any damage.

\section{The ISS-CREAM Instrument}

The basic instrument flown on the CREAM balloon craft had to be repackaged into the smaller volume available on the Japanese Experiment Module Exposed Facility (JEM-EF) for the ISS-CREAM instrument [7]. The ISS-CREAM instrument is configured with the CREAM calorimeter [6] including carbon targets for energy measurements and four layers of a finely segmented Silicon Charge Detector [8] for charge measurements. Multiple layers provide better charge resolution and increase the redundancy for longer operation. These detectors have already demonstrated their capabilities to determine the charge and energy of high-energy cosmic rays from $10^{10}$ to $>10^{14} \mathrm{eV}$ for the proton to iron elemental range with excellent resolution [3].

In addition, two new compact detectors have been developed: Top/Bottom Counting Detectors (TCD/BCD) and Boronated Scintillator Detector (BSD). The TCD and BCD each consist of a plastic scintillator and 400 photodiodes to provide electron/hadron separation using lateral and longitudinal shower profile difference between electrons and hadrons. TCD is located between the instrument's carbon target and the calorimeter, and the BCD is located below the calorimeter as shown in Fig. 1. They also provide a redundant cosmic ray trigger. Details of the TCD/BCD design and measured performances are presented elsewhere [9-11].

The BSD is configured with a boron loaded plastic scintillator (5\% boron concentration by weight and the natural ${ }^{10} \mathrm{~B}$ abundance of $20 \%$ ) and 18 photo multiplier tubes. It is located below BCD under the calorimeter to measure the late (>400 ns) thermal neutron shower activity by detecting the boron capture of these thermal neutrons. Hadron-induced showers tend to be accompanied by significantly more neutron activity than are electromagnetic showers. The hadron rejection power derived from TCD/BCD can be significantly enhanced by making use of the thermal neutron measurements with the BSD. Results from a beam test and the expected performance are discussed in another paper [12].

The scope of work required for the ISS investigation includes modification of instrument components for the ISS environment, in addition to assessing safety and mission assurance concerns. The instrument must be functionally tested and qualified to meet the launch vehicle and on-station requirements for operations on the ISS. The instrument has been repackaged within a structure that meets the JEM-EF interface requirements. While the basic design of the instrument is mature, and it has heritage operating over many years in the near-space environment, the radiation effects on electronic circuits had to be adequately addressed for ISS-CREAM. Components were selected and utilized in a manner to prevent the possibility of failures as a result of Single Event Latch-up (SEL), and to assure that Single Event Upset (SEU) and Single Event Transient (SET) effects will have minimal impact on data collection [13]. 
The ISS-CREAM payload is about the size of a refrigerator (see Fig. 1) with 1,300 kg mass, including government furnished equipment such as grapple fixtures and the Payload Interface Unit (PIU). The estimated $\sim 600 \mathrm{~W}$ power and nominal data rate of $350 \mathrm{kbps}$ are all within the available JEM-EF resources. ISS-CREAM utilizes an Active Thermal Control System, a Fluorinert fluid loop, provided by the JEM-EF through the standard PIU.

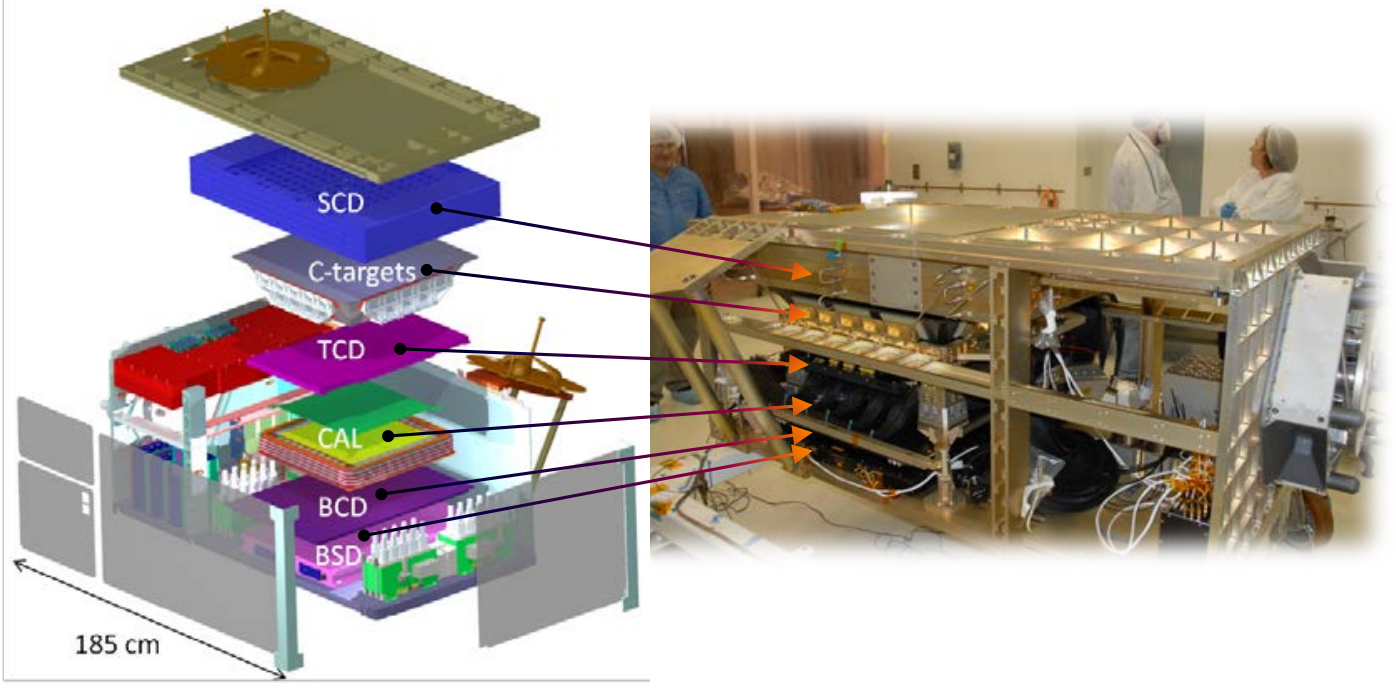

Figure 1: The ISS-CREAM instrument drawing (left) and a photo of the actual payload during the integration (right).

\section{Status and Plan}

The ISS-CREAM proposal was submitted to NASA in 2011. Following the Instrument Preliminary Design Review and the Launch Vehicle Accommodation Study in 2012, the Critical Design Review (CDR) was completed in 2013. The Phase II Safety Review with JSC's Payload Safety Review Panel and a Technical Interchange Meeting (TIM) with JAXA were also completed in 2013. All of the detector siubsystems were delivered to NASA for integration after the mechanical qualification and thermal vacuum tests to meet the launch vehicle and on-orbit requirements for operations on the ISS. Following the Systems Integration Review (SIR), integration of the payload at NASA GSFC WFF started in 2014. The fully integrated payload was delivered to NASA GSFC for the system level qualification in early 2015. The Electromagnetic Interference (EMI) testing has been completed to ensure Electromagnetic Compatibility (EMC) with the ISS. Following EMI/EMC and power quality tests, including radiated susceptability and conducted emmisions, the payload is currently undergoing mechanical verification including vibration, sine burst, and accoustics. After the thermal vacuum tests, the payload will be launched from NASA Kennedy Space Center on SpaceX to be installed on the ISS JEM-EF.

In parallel with the hardware development, the software has been developed. The instrument interface with the ISS is Ethernet based, as was the Command and Data handling Module for the CREAM balloon flights. The ISS-CREAM operation will be the same as CREAM, except the Huntsville Operations Support Center (HOSC) Payload Operations Integration Center (POIC) at 
NASA Marshall Space Flight Center (MSFC) will replace NASA GSFC WFF's Engineering Support Center. The CDAQ has been modified to interface with the POIC using the Telescience Resources Kit (TReK http://trek.msfc.nasa.gov). A significant rewrite of software was avoided through use of the Software Toolkit for Ethernet Lab-Like Architecture (STELLA) developed by the Boeing Company. The CDAQ handles command and data both in real time and in playback. The command uplink and data downlink will be through the Tracking and Data Relay Satellite System (TDRSS), exactly analogous to CREAM balloon flight operations.

The CREAM team has extensive experience in running the Science Operation Center (SOC) at the University of Maryland (UMD) for 6 balloon flights over a period of 6 years. The SOC has been configured to provide secure data links with the POIC; adequate data processing hardware; and the data archive. As the raw data sets are piped to the SOC, backup copies will be made mmediately and stored on our RAID system in the UMD Research Data Center (RDC). The expected raw data volume is about $1.3 \mathrm{~TB} /$ year, $\sim 4 \mathrm{~TB}$ for 3 years, and the reconstructed data is about 4 TB/year, 12 TB for 3 years. The existing Internet bandwidth between MSFC and the SOC averages $4 \mathrm{Mbps}$, which is sufficient to transmit the data.

The necessary operating systems and software, including CDAQ and STELLA, were installed on the server system, Science Flight Computer (SFC), and client computers for the ground operations. A remote connection test between SOC and HOSC was completed successfully. To meet MSFC's requirements, development testing of the CDAQ software system was conducted at the Space Systems Integration and Test Facility (SSITF) at MSFC by taking the SFC to the SSITF. The actual Command and Data Handling (C\&DH) setup on the ISS was simulated by connecting the ISS-CREAM SFC to the Payload Rack Checkout Unit (PRCU). During the testing, reliable flow of commands and telemetry between HOSC and the UMD SOC were established. The End-to-End tesing including the payload at GSFC and PRCU was recently completed.

ISS-CREAM will measure the energy spectra and composition of cosmic rays to the energies capable of generating gigantic airshowers which have been observed mainly on the ground, thereby providing overlaps with the ground based indirect measurements. This experiment will provide unique data for studying the origin of cosmic rays and the mechanism by which they are accelerated to characterize the mechanism of cosmic-ray acceleration with far greater precision than heretofore possible. An order of magnitude increase in data collecting power is possible by utilizing the ISS to reach the highest energies practical with direct measurements. The long exposure above the atmosphere offers orders of magnitude greater statistics without the secondary particle background inherent in balloon experiments investigating the origin of cosmic rays. This mission above the atmosphere offers the opportunity to augment the science reach of CREAM, i.e., judicious selection of ancillary detectors that fit within the restricted JEM-EF volume can add sensitivity to cosmic-ray electrons, which are of great current interest in particle astrophysics.

\section{Acknowledgements}

The authors thank NASA GSFC WFF for project management and engineering support, and NASA JSC ISS Program Office for launch support and the ISS accommodation. This work was supported in the U.S. by NASA grants NNX11AC52G, NNX08AC15G, NNX08AC16G and their predecessor grants, as well as by directed RTOP funds to NASA GSFC WFF. It is supported in Korea by the Creative Research Initiatives of MEST/NRF and by National Research Foundation 
Grants NRF-2014R1A2A2A01002734, NRF-2014R1A1A2006456. It is supported in France by IN2P3/CNRS and CNES and in Mexico by DGAPA-UNAM and CONACYT. The authors also thank H. S. Choi, Korea Institute of Industrial Technology, for contributions to the SCD termal vacuum tests, M. Geske, Penn State, for contributions to the BSD, and M. A. Coplan, University of Maryland, contributions to CAL electronics vacuum tests.

\section{References}

[1] The National Research Council Decadal Study Report, Astronomy and Astrophysics in the New Millennium, Washington DC: The National Academies Press, (2001)

[http://www.nap.edu/openbook.php?record_id=9839].

[2] H. S. Ahn et al. The Cosmic Ray Energetics And Mass (CREAM) instrument, Nucl. Inst. Meth. A, 579 (2007) 1034 [http://dx.doi.org/10.1016/j.nima.2007.05.203].

[3] H. S. Ahn et al., Discrepant Hardening Observed in Cosmic-ray Elemental Spectra, Astrophys. J. Lett. 714 (2010) L89 [doi:10.1088/2041-8205/714/1/L89].

[4] S. Nam et al., Performance of a Dual Layer Silicon Charge Detector During CREAM Balloon Flights, IEEE Trans. Nucl. Sci., 54/5 (2007) 1743.

[5] M. Buénerd, et al., A Cherenkov imager for the charge measurement of Nuclear Cosmic Rays in the CREAM II instrument, Proc. 29 $9^{\text {th }}$ Int. Cosmic Ray Conf., Pune, 3 (2005) 277.

[6] M. H. Lee et al., The CREAM-III calorimeter, Proc. 30th Int. Cosmic Ray Conf., Merida, 2, (2007) 409.

[7] E. S. Seo et al, Cosmic Ray Energetics And Mass for the International Space Station (ISS-CREAM), Advances in Space Research, 53/10 (2014) 1451 [doi: 10.1016/j.asr.2014.01.013].

[8] J. Lee et al. Design, Fabrication and Performance of the Silicon Charge Detector for the ISSCREAM, this conference (2015) ID 1349.

[9] H. J. Hyun et al, Performances of photodiode detectors for top and bottom counting detectors of ISS-CREAM experiment, Nucl. Inst. Meth, 787 (2015) 134 [doi:10.1016/j.nima.2014.11.075].

[10] Y. S. Hwang et al., Construction and Testing of a Top Counting Detector and a Bottom Counting Detector for the Cosmic Ray Energetics And Mass Experiment on the International Space Station, J. of Instrum. (2015) in press.

[11] J. M. Park et al. Top and Bottom Counting Detectors for the ISS-CREAM experiment, this conference (2015) ID 433.

[12] J. T. Link et al. Design and Performance of the ISS-CREAM Boronated Scintillator Detector, this conference (2015) ID 659.

[13] Y. Amare et al., Study of radiation effects on CREAM electronics, Proc. 33rd Int. Cosmic Ray Conf., Rio de Janeiro, Brazil (2013) ID 630. 